\title{
Design and analysis of DOE-based microinterferometer
}

\author{
Michał Józwik, Krzysztof Wielgo, and Tomasz Kozacki \\ Institute of Micromechanics and Photonics, Faculty of Mechatronics, Warsaw University of Technology, \\ 8 Św. A. Boboli St., 02-525 Warsaw, Poland
}

Received September 10, 2011; accepted September 26, 2011; published September 30, 2011

\begin{abstract}
This paper describes the design and numerical study of a Twyman-Green microinterferometer based on Diffractive Optical Elements (DOEs). Its compact design allows to fabricate several interferometers in an array-like pattern on an optical wafer - with horizontal dimensions appropriately adapted to the tested structures. To prove the design concept of the proposed interferometer configuration a laboratory setup was realized. The first results of the interferometric measurements on an MEMS object are presented.
\end{abstract}

The industry of massive microsystem production requires fast, high quality, nondestructive measurements of active and passive parameters of fabricated elements. In the frame work of the EU project SMARTIEHS [1] a new approach towards the parallel inspection of such devices at wafer level has been developed. The optical unit consists of two arrays of microoptical interferometric sensors: Mirau type low coherence interferometers and DOE-based laser interferometers in the Twyman-Green (TG) configuration [2]. It is presented that with diffractive optical elements (DOEs) it is also possible to design an interferometer with similar functionality as the one based on standard optical components. DOEs offer unique features such as high integration and compactness [3-4].

In this paper we propose an optional configuration of the integrated TG interferometer based on diffractive gratings. The scheme of the device is presented in Fig.1.

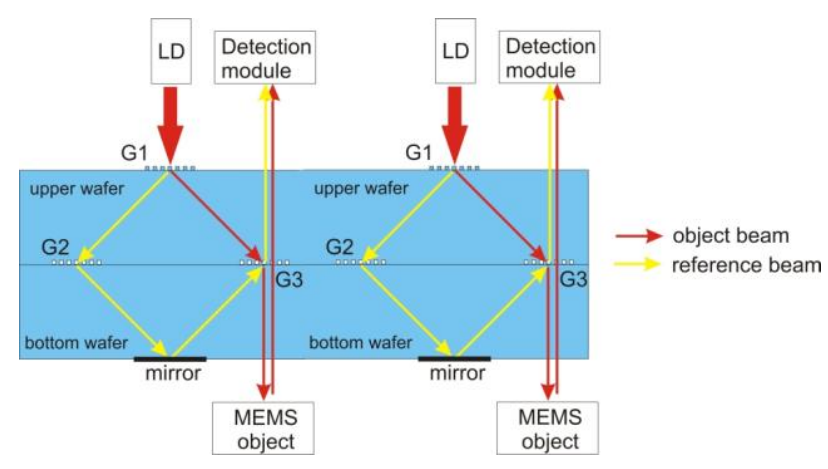

Fig. 1. Scheme of proposed microinterferometer.

It consists of two $\mathrm{SiO}_{2}$ wafers bonded together, the upper with one and the bottom with two diffraction gratings. The grating G1 is illuminated by a collimated laser diode LD beam and acts as a beam splitter creating two beams in -1 and +1 diffraction order. The grating G2 and a mirror direct first beam in the reference arm. The grating G3 is the key element of the interferometer. The grating G3 directs the second beam to an MEMS object under test and after reflection from the object's surface combines the object and reference beam together. An interferogram is imaged by the detection module.

For design and numerical study we have implemented and then applied the best suited method that meets our needs and seems to be very appropriate for binary gratings - Rigorous Coupled Wave Theory [5-8] with implementation of the algorithm of the Enhanced Transmittance Matrix Method [5]. Results were obtained for both TE and TM polarizations, however here we show only the TE case. Using the developed RCWA software, we have optimized all binary gratings in interferometer design. The optimization process has to take into account limits imposed by element geometry and possibility of technological realization. The geometrical constraint states that the aspect ratio of the structures should be smaller than 10 [9]:

$$
a=\min \left(\frac{k \Lambda}{d}, \frac{(1-k) \Lambda}{d}\right)<10,
$$

where: $k$ - the fill factor, $d$ - the grating depth, $\Lambda$ - the grating period. We preferred smaller values of depths and fill factors around 0.5 .

Let's first consider grating G1. The grating deflects a normally incident planar wavefront. The angle of the first diffraction order is governed by the grating equation. For illumination wavelength $\lambda=658 \mathrm{~nm}$ and air- $\mathrm{SiO}_{2}$ interface $\left(\mathrm{n}_{\mathrm{SiO} 2}=1.457,[5]\right)$ it equals $26.85^{\circ}$. At gratings $\mathrm{G} 2$ and $\mathrm{G} 3$ this angle is illuminated. Additionally, grating G1 serves as a beam-splitter. Light impinges at the normal angle and should be split into two beams with equal and high intensities. The efficiency map shown in Fig. 2 points out that the highest efficiency of $\eta=0.445$ is received with $d=0.75 \mu \mathrm{m}$ and $k=0.325$. These parameters fulfil constraint (1) as well.

Grating G2 has to change the direction of the incident beam - from $\alpha_{\text {inc }}=-26.85^{\circ}$ to $\alpha_{\text {diffr }}=+26.85^{\circ}$ with high 
efficiency. Therefore the Bragg grating should be used. The Bragg grating condition [7] gives a period $\Lambda=0.5 \mu \mathrm{m}$. Light after passing the upper wafer impinges on the grating filled with air and gets transmitted into $n=+1$ st order with high efficiency.

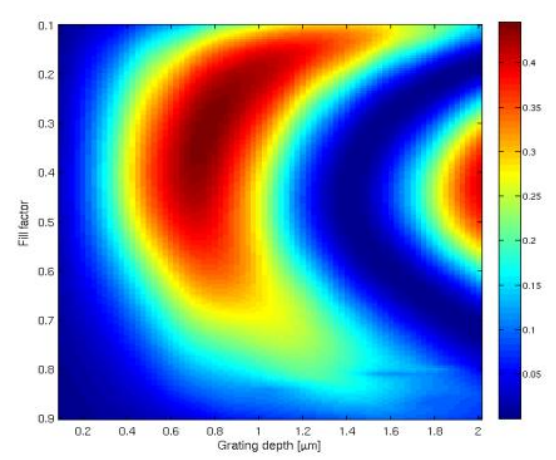

Fig. 2. Efficiency map for the grating G1; dependence on grating depth and fill factor.

The efficiency of grating G2 is shown in Fig. 3, where one can find optimum efficiency achieved for $d=1.05 \mu \mathrm{m}$ and $k=0.54$ reaches almost $\eta=1$.



Fig. 3. Efficiency map for the grating G2; dependence on grating depth and fill factor.

Grating G3 is the key element of a DOE-based interferometer. The object beam passes twice through the element and the reference beam - once. In the case of the reference beam, light impinges the grating at angle $\alpha_{\text {inc }}=+26.85^{\circ}$ and is transmitted into $n=-1^{\text {st }}$ order $\left(\alpha_{\text {diffr }}=0^{\circ}\right)$. The object beam is impinging grating G3 at $\alpha_{\text {inc }}=+26.85^{\circ}$ and is transmitted into $n=-1^{\text {st }}$ order directed normally to the object. Then the object beam is reflected from measured object surface and is simply transmitted by G3 (incidence angle is $\alpha_{\mathrm{inc}}=0^{\circ}, \mathrm{n}=0^{\text {th }}$ order, $\alpha_{\mathrm{diffr}}=0^{\circ}$ ). The selection of optimal parameters is not so obvious for element G3. We need to consider efficiencies of reference and object beams together. In an interferometer we want high contrast and high intensity of light arriving at the detector (expressed by efficiencies of both arms of an interferometer). We notice that even for perfectly reflective object efficiency for an object beam it is much weaker than for a reference one. Therefore we look for parameters that give the highest efficiency for an object arm. This happens, when $k=0.38$ and $d=1.1 \mu \mathrm{m}$ (Fig. 4). In this case reference beam is still stronger $\left(\eta_{\text {object }} \approx 2.3 \%\right.$ when $\eta_{\text {reference }} \approx 8.7 \%$ ).

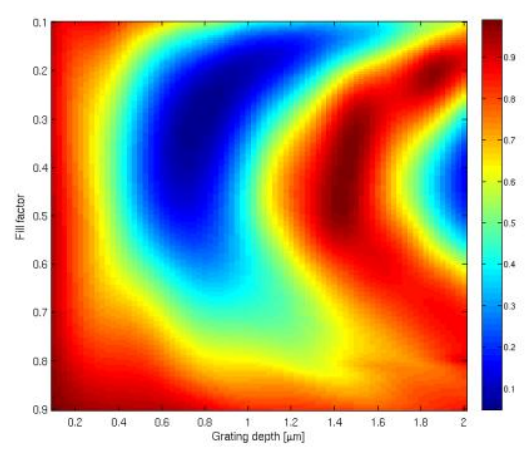

Fig. 4. Efficiency map for the third grating (G3); dependence on grating depth and fill factor; $\alpha_{\text {inc }}=+0^{\circ}$, transmission into $\alpha_{\text {diffr }}=+0^{\circ}$.

To get maximum contrast, we need to decrease the reference arm efficiency. It can be obtained by change of G2 grating parameters $\left(\eta_{\text {Bragg }}=26.5 \%\right.$ for $(k=0.51$, $d=0.35 \mu \mathrm{m})$ or $(k=0.42, d=1.55 \mu \mathrm{m}))$. Equivalently, instead of the Bragg grating we can use grating with a period $\Lambda=1 \mu \mathrm{m}$. Then of our interest is $n=+2^{\text {nd }}$ transmitted order. For this grating $\eta=26.5 \%$ for $\left(\mathrm{n}=+2^{\mathrm{nd}}\right.$ order, $k=0.51$, $d=0.8 \mu \mathrm{m})$ (Fig. 5 ). In both cases, $\eta_{\text {reference }} \approx \eta_{\text {object }} \approx 2.3 \%$ and overall efficiency at detector would be $4.6 \%$ only but contrast reaches unity.

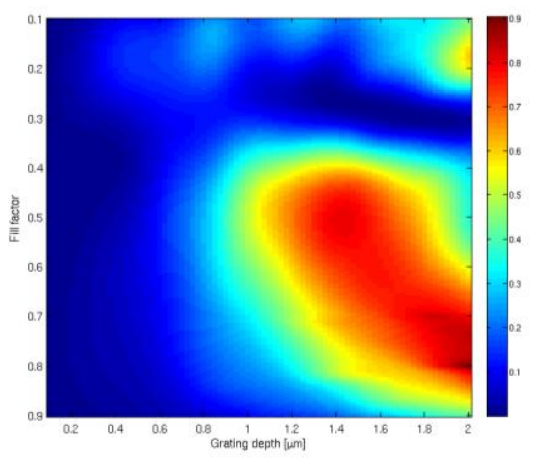

Fig. 5. Efficiency map for the grating G2; dependence on grating depth and fill factor; $\alpha_{\mathrm{inc}}=+26.85^{\circ}$, transmission into $\alpha_{\text {diffr }}=-26.85^{\circ}$.

In order to prove the design concept of the proposed interferometer configuration a laboratory setup was realized. We assembled the setup as in Fig. 6. As a laser source, a laser diode Hitachi HL6535MG $(\lambda=658 \mathrm{~nm}$, power $80 \mathrm{~mW}$, single mode) with a temperature controller (Thorlabs TED 200C) and an LD driver (Thorlabs LDC 205C) was used. Additionally, the laser diode worked in pulse mode allowing to decrease disturbing interference effects. The plane illumination wavefront was obtained 
with a collimating lens $(f=200 \mathrm{~mm})$. Grating $\mathrm{G} 2$ and the metallic mirror layer from the design (Fig. 1) were replaced with optical mirrors. Gratings G1 and G2 are the same, and optimized for $\lambda=650 \mathrm{~nm}$ to diffract into $+/-1 \mathrm{st}$ order. These high quality binary diffraction elements were provided by Fraunhofer IOP, Jena, Germany. Gratings have height of $\mathrm{h}=100 \mathrm{~nm}$ and the period of $1 \mu \mathrm{m}$.



Fig. 6. Laboratory setup.

The detector used to capture the interference pattern is a CCD camera CV-A1, JAI Company. In setup imaging of the object and an interference pattern is produced with a so-called $4 \mathrm{f}$ setup, with a spatial filter (SF2) in the Fourier plane. The magnification of the described setup is $M=1 \mathrm{x}$.

The results presented in Fig. 7 are satisfactory. Although gratings are not optimized for transmission into $0^{\text {th }}$ order, the quality of fringes, intensity and contrast $(>0.7)$ are reasonable and sufficient. This means that interferogram analysis methods (such as Phase Shifting Interferometry or Fourier Analysis) may be applied. The fringes quality allows for further processing of the interference pattern in order to find the shape of a deflected MEMS membrane on the wafer. The major problem of the presented laboratory setup was the lack of properly designed grating G3, which resulted in a low intensity object beam Therefore, in order to improve overall interferometer performance, this grating should be replaced by an optimized element.

Concluding, we reached the goal of compact microinterferometer design based on diffractive optical elements that could be fabricated in a matrix on the optical wafer. Using the developed RCWA tool, the parameters of three diffraction gratings used in the design of the interferometer were proposed and optimized. Finally, the proof of the principle of the interferometer functionality containing diffraction gratings was demonstrated on an experimental laboratory setup. (a)

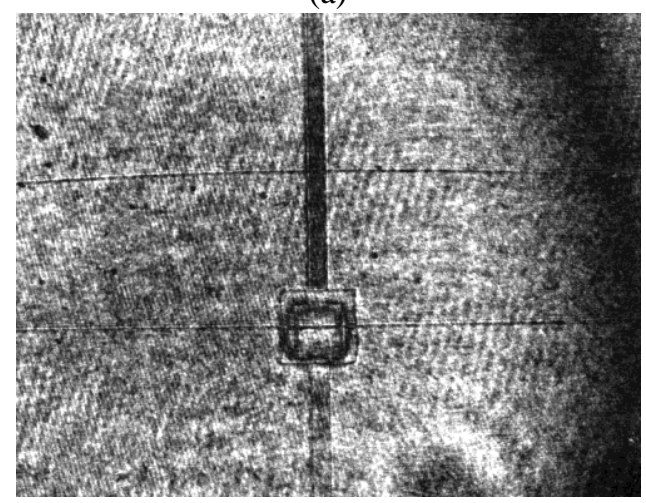

(b)

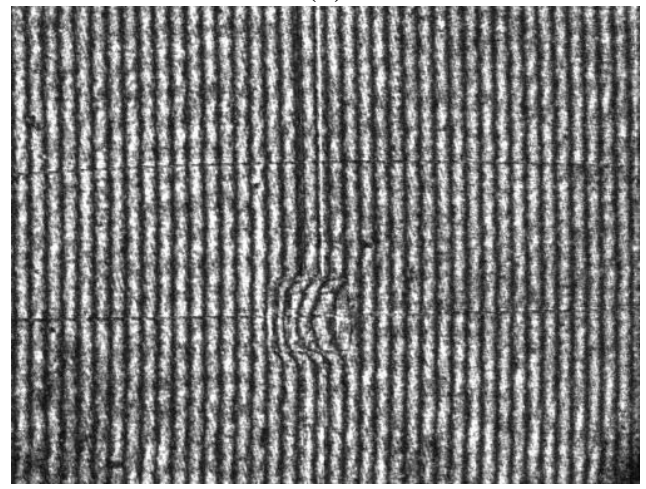

Fig. 7. Interferograms for: a) uniform field detection, b) tilted object.

This work has been realized within the SMARTIEHS collaborative project, funded under the Grant Agreement 223935 to the $7^{\text {th }}$ Framework of the European Union.

The development of RCWA was supported by the statutory funds of the Warsaw University of Technology. The authors thank Dr. R.C. Rumpf for his great help in the RCWA implementation.

\section{References}

[1] http://www.ict-smartiehs.eu

[2] M. Kujawinska et al., Proc. SPIE 7791, 779103 (2010).

[3] T. Kozacki et al., Opt. Exp. 17, 13758 (2006).

[4] B. Kim et al., J. Manuf. Sci. Eng. 126, 807 (2004).

[5] R.C. Rumpf, PhD Thesis, College of Optics / CREOL\&FPCE (2006).

[6] M.G. Moharam et al., J. Opt. Soc. Am. A 12, 1068 (1995).

[7] P. Lalanne, M. Morris, J. Opt. Soc. Am. A 13, 779 (1996).

[8] V.A. Soifer, Methods for computer design of diffractive optical elements (New York, John Wiley \& Sons 2002).

[9] D. Dias et al., J. Opt. A 3(3), 164 (2001). 